\title{
Chronique bibliographique. Lectures et usages de Foucault
}

Review essay. Readings and uses of Foucault

Reseña bibliográfica. Lectura y uso de Foucault

\section{Rémi GUITTET}

\section{(2) OpenEdition \\ Journals}

\section{Édition électronique}

URL : http://journals.openedition.org/conflits/17386

DOI : $10.4000 /$ conflits. 17386

ISSN : $1777-5345$

Éditeur :

CCLS - Centre d'études sur les conflits lilberté et sécurité, L'Harmattan

Édition imprimée

Date de publication : 20 décembre 2008

Pagination : 137-144

ISBN : 1157-966X

ISSN : 1157-996X

\section{Référence électronique}

Rémi GUITTET, «Chronique bibliographique. Lectures et usages de Foucault », Cultures \& Conflits [En ligne], 72 I hiver 2008, mis en ligne le 19 mai 2009, consulté le 30 mars 2021. URL : http:// journals.openedition.org/conflits/17386 ; DOI : https://doi.org/10.4000/conflits.17386 


\title{
Chronique bibliographique. Lectures et usages de Foucault
}

\section{Rémi GUITTET}

Rémi Guittet est élève à l'Ecole normale supérieure de Paris et lecteur à l'université de Leipzig où il tient un séminaire d'histoire culturelle. Il poursuit par ailleurs ses études en philosophie antique et politique à l'université Paris-I.

A propos de :

Veyne P., Foucault. Sa pensée, sa personne, Paris, Albin Michel, 2008, 215 p.

Artières P., Potte-Bonneville M., D’après Foucault, Paris, Les Prairies ordinaires, 2007, $375 \mathrm{p}$.

\begin{abstract}
en croire ses propres mots, Foucault rêverait de disparaître et de ne plus

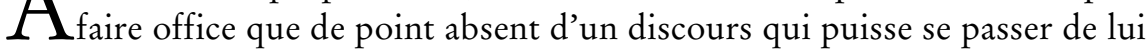
et le laisser hors-champ ${ }^{1}$. Si tout discours, et éminemment un discours de professeur au Collège de France, est en effet un discours de pouvoir, on comprend que la position critique soit contrainte de tendre vers l'anonymat, nom transitoire de la disparition. Disparaître, se laisser traverser par le discours, et non l'investir d'un pouvoir, en faire le lieu de la critique sans en faire le lieu d'un nouvel ordre, voilà le but idéal, au sens fort (il est régulateur et non atteignable en tant que tel), vers lequel tend dans la pratique la critique foucaldienne. En quoi consiste-t-elle ? Refuser les discours qui utilisent des essences universelles comme principes régulateurs. Refuser l'insistance du pouvoir qu'ils représentent. Les contourner pour en extirper la position archéologique, l'implicite normativité qu'ils véhiculent et construisent tout à la fois. Contre eux, privilégier le "spécifique ", ce qui dans un endroit donné se réalise et touche les acteurs qui y prennent part, et qui n'est pas à penser en référence à un système d'interprétation préétabli, autrement dit une norme, ou des universaux, mais au contraire comme l'inédit qui ne s'y laisse pas réduire.
\end{abstract}


Pourtant, il est bien impossible au moment d'ouvrir le dossier Foucault de taire l'homme. Bien au contraire, l'homme agissant, combattant, loquace même, ne cesse d'apparaître dans les mémoires et les imaginations. Si bien que l'on découvre très vite un hiatus dans l'approche (de l'œuvre) de Foucault que l'on pourrait énoncer abruptement ainsi : comment étudier Foucault sans parler de Foucault? Avec Foucault, en effet, il faut toujours faire l'exercice de savoir ce que l'on fait de la « boîte à outils ». Lire Foucault, c'est à chaque fois, par et à travers le geste même de la lecture, être confronté à une exigence de repositionnement et de remise en question de ses pratiques universitaires, militantes, érudites, ou tout simplement privées. Le texte de Foucault ne laisse jamais en repos : il appelle à se fondre en lui dans le même temps qu'il réclame pour son propre compte le statut de coup d'essai, de tentative. Discours de maître qui s'en dédit...

Aspect notable que Baudrillard a pu prendre en grippe, et dont il réclamait le dépassement (à la mode hégélienne), autrement dit, l'oubli après assimilation ${ }^{2}$. Si Foucault parle si bien du pouvoir et des discours de pouvoir, comment ne pas, en toute légitimité, s'inquiéter du sien ? L'inquiétude certes, pour le lecteur de Foucault, ne saurait être celle de l'institution, celle que Mathieu Potte-Bonneville nomme si bien «la sagesse soncieuse des magistrats $^{3}{ }^{»}$. C'est une inquiétude d'usager : que faire de Foucault ? Réceptionner l'œuvre de Foucault est inséparable du geste qui le récupère, et ce en un sens éminent : comment envisager le commerce et le débat autour d'un penseur qui s'est acharné à ne pas constituer sa pensée en école, à privilégier le «spécifique » à l'universel, à exposer des pistes et des méthodes plutôt que des résultats ou des thèses sonnantes et trébuchantes ? La question n'est pas triviale : il s'agit de savoir ce que l'on peut faire de, aussi bien qu'avec Foucault. Ou encore, qu'est-ce qu'un auteur, ou un acteur, «foucaldien » ? Deux ouvrages et trois auteurs s'engagent dans une réponse.

Parmi les publications récentes touchant au foucaldisme, deux livres partagent une communauté et une divergence de perspective fécondes. Foucault. Sa pensée, sa personne, de Paul Veyne, ainsi que D'après Foucault, recueil d'études et allocutions réécrites pour l'occasion par Philippe Artières et Mathieu Potte-Bonneville ${ }^{4}$. Le premier de ces ouvrages touche par de multiples côtés au genre de l'éloge funèbre à un ami. Paul Veyne cherche à redonner un souffle vivant à la pensée de Foucault en se servant à la fois des textes et du personnage lui-même, deux sujets qu'il connât bien, pour avoir été lecteur aussi bien qu'ami de l'intéressé. Le ton y prend souvent l'allure d'un plaidoyer

2. Baudrillard J., Oublier Foncault, Paris, Ed. Galilée, coll. « L'espace critique », 1977, pp. 9-12.

3. L'expression se trouve dans: Potte-Bonneville M., in D'après Foucault, Paris, Les Prairies ordinaires, 2007, p. 224.

4. Veyne P., Foucault. Sa pensée, sa personne, Paris, Albin Michel, 2008, 215 p. ; Artières P., Potte-Bonneville M., op. cit. 
en faveur du "samouraï ${ }^{5}$ ", ce "mince, élégant et tranchant personnage». Voulant renverser les idées reçues sur son ami, il s'attache à lui restituer sa «spécificité» propre, face à tous les «universalismes» qui ont pu vouloir refaire un Foucault à leur convenance, avance-t-il. Le second ouvrage tranche quant à lui d'emblée le problème de l'héritage, ou plutôt, il fait de l'héritage son noyau : "Nous n'avons pas connu Foucault ", proclament dès la première page de cet ouvrage non dédicacé les deux co-auteurs. Alternant perspectives historique et philosophique, Philippe Artières et Mathieu Potte-Bonneville manipulent sans cesse les objets de la critique foucaldienne pour en extraire soit de nouvelles interprétations, soit des pistes nouvelles dans le champ politique ou érudit. D'un côté donc, Paul Veyne s'attache à restituer une pensée, et de l'autre, Artières et Potte-Bonneville s'attachent à discuter un héritage et à le bousculer en s'en servant copieusement, non sans le faire savoir.

Y aurait-il un éternel paradoxe à être disciple de Michel Foucault ? La question taraude explicitement Artières et Potte-Bonneville : comment être les continuateurs d'un maître évasif, presque sournois, en tout cas ironique ? C'est sans doute ce problème qui a conduit les auteurs à mêler aux concepts l'histoire, aux préceptes les exemples. Cette alternance entre des articles à tendance historique et d'autres à tendance conceptuelle permet aux deux auteurs d'indiquer divers «points de problématisation 6 » qui s'attachent à relier les pratiques et les concepts foucaldiens dans un dialogue permanent. Philippe Artières, dirigeant du centre Michel Foucault où sont conservés la plupart des documents relatifs à la vie et au travail du philosophe, fait en effet ici œuvre d'historien et d'archiviste : de multiples monographies centrées sur les activités du GIP 7 , ainsi que de nombreuses analyses d'anthropologie de l'écriture basées sur les thèses de Foucault, ou encore des comptes rendus d'exploitation des archives du centre Michel Foucault (la prise de parole chez Foucault, analyse de l'évolution du travail de Foucault après son passage par l'archive, etc.) forment les diverses parties de sa contribution. Les interventions de Mathieu Potte-Bonneville relèvent quant à elles d'un registre plus conceptuel et « philosophique », puisque l'auteur s'attache par exemple à éclairer les concepts foucaldiens par des concepts deleuziens ${ }^{8}$, à dégager la notion de droit chez Foucault des notions philosophiques classiques (problème de la norme et problème du fondement), ou encore à rapporter les notions de «souci de soi » tant aux problématiques stoïciennes anti-

5. C'est en effet ainsi que l'auteur avait pensé primitivement intituler l'ouvrage : « Le samouraï et le poisson rouge ", comme il est dit p. 11.

6. Comme M. Potte-Bonneville le montre p. 232 à propos de la rencontre, sur le terrain du droit, de la rationalité juridique et de la rationalité normative, autrement dit, des exigences de fondement de jure et des exigences pratiques de la « gouvernementalité ».

7. Le Groupe d'information sur les prisons (GIP), que Michel Foucault, de concert avec JeanMarie Domenach, Pierre Vidal-Naquet et d'autres, fonde en février 1971. Il s'agissait de soutenir des révoltes de prisonniers visant les conditions de l'emprisonnement, et non la dénonciation pure et simple de la prison comme répression.

8. La comparaison est fréquente, comme c'est le cas dans les chapitres «Enseigner», « Disparaître », «Contrôle ». 
ques ${ }^{9}$ qu'aux questions d'éthique de la modernité telles qu'elles sont abordées aujourd'hui par Taylor ou Rancière ${ }^{10}$. Par là, les auteurs entendent faire acte de « reprise ». Reprise de ce qui est identifié comme les "gestes », «luttes » et «programmes » 11 de Foucault.

L'introduction (seul texte cosigné de l'ouvrage) présente dans un style vif et alerte les enjeux du livre : porter aussi loin que possible les armes de la critique spécifique. S'il faut reprendre Foucault, c'est que son travail est précisément un travail qui se joue au corps à corps avec les événements, qui est incessamment modifié par eux, en ce sens que les pensées à prétention universelle cachent bien plus des dispositifs de pouvoirs et de coercition que d'hypothétiques puissances de vérités, axiome méthodique foucaldien que les auteurs prennent à leur compte. C'est pourquoi l'ouvrage résonne toujours de la bataille qui se joue derrière les textes. A un concept succède toujours une action : le récit d'une prise de parole, d'une lutte, de la rédaction d'un manifeste, d'un soulèvement pénitentiaire, ou d'une organisation de lutte contre le SIDA. La critique foucaldienne doit être une critique en prise avec le moment, réorganisable et fluide, convoquée par l'histoire et non en retrait sur elle. C'est pourquoi l'histoire répond à la philosophie, l'événement appelant la critique, c'est-à-dire les armes pour l'action, ce dont les auteurs ne se cachent pas. Les chapitres les plus solides conceptuellement décrivent sous de multiples angles les implications de la démarche critique. Comment la nécessité du diagnostic conduit au corps, à la déprise, mais, comme par contrecoup, dans le parcours de Foucault, aussi au «sonci de soi». Ces chapitres repèrent un parcours, ce que les auteurs appellent, non sans raison, des « gestes ». Le geste foucaldien ressort à plusieurs catégories cohérentes de manière interne aussi bien qu'entre elles. Il y a le "geste philosophique », celui par lequel Foucault demeure un continuateur d'une longue tradition qui se reconnait sous ce terme. Mathieu Potte-Bonneville l'identifie à une nouvelle expérience du stoïcisme. Celle-ci relève, comme l'antique, de la discipline et de l'ascèse. Comme le stoïcien (qui rejoint en cela le sceptique), Foucault commence par suspendre son jugement. Or, cet exercice est aussi bien érudit que pratique : c'est une méthode de recherche qui contient les bases d'une disposition ascétique et d'une « vision du monde » (Weltanschaunng) telle que la philosophie les affectionne. Le stoïcien antique y cherchait l'ataraxie. Foucault, lui, y a trouvé, selon Potte-Bonneville, la place pour un nouveau rapport à soi : la déprise. Si en effet la critique des rapports de pouvoir se veut radicale, elle doit faire l'épreuve d'un détachement; c'est ce que Veyne nomme la «sortie du bocal », le bocal figurant le monde où circulent les vérités et les hommes. Ce geste philosophique est solidaire d'une position et d'un geste politiques : aucune vérité

9. In «Enseigner».

10. In «Ethique».

11. Ce sont les trois parties qui divisent l'ouvrage. Elles comportent respectivement 7, 6 et 2 chapitres. 
ne gouverne en tant que telle, mais seulement des effets de vérités, c'est-à-dire de pouvoir. C'est là que se cachent les « universalismes », ces machines intégratrices qui broient sous leur poids le monde de la vie, autre manière de nommer ce «spécifique» dont parlent aussi bien Veyne qu'Artières et PotteBonneville. Par conséquent, le plus sage est d'inventer, de recourir à de nouvelles formes de luttes, puisque la politique n'est qu'un champ de bataille, celui des rapports de pouvoir, que les dispositifs de savoir appuient. Ces conclusions sont abondamment illustrées dans l'ouvrage. Ainsi, la seconde partie, consacrée aux « luttes », fait-elle la part belle aux soulèvements de prisonniers et à la manière de les penser comme luttes d' " usagers », ou encore aux récits relatifs à l'association AIDES, fondée par Daniel Defert. Les chroniques sont toujours précises et documentées, et permettent à la pensée théorique de s'incarner dans des modalités pratiques. Les mots d'ordre des auteurs en cette matière sont : écoute, inventivité, création. Pour eux, Foucault est celui qui a appris à faire voir ce qui est train de se passer, à ne pas considérer les événements comme des manifestations de structures ou de concepts bien connus. L'événement est ce qui doit déranger la pensée et lui apporter sa vie.

Telle est la manière de reprendre l'héritage foucaldien qu'Artières et Potte-Bonneville jugent «fidèle ». La fidélité ici doit s'entendre comme cette probité qui appelle le commentaire, l'interrogation et l'invention, par opposition à la répétition scolastique ou académique. C'est en ce sens que Foucault est un maître stoïcien aux yeux de Potte-Bonneville : il apprend par son geste critique même, en place d'enseigner des thèses toutes faites. Certes, dira-t-on. Cependant, pourquoi avoir alors toujours besoin de Foucault ? Pourquoi sans cesse revenir sur les thèmes de Foucault et, finalement, sur les thèses de Foucault, c'est-à-dire ses conclusions critiques ? Une conclusion critique est par définition provisoire ; dès lors, que penser des nombreuses répétitions du livre 12 ? On ne saurait accuser les deux auteurs de tomber dans le dogmatisme. Toutefois, il est frappant de remarquer combien les exemples qui soutiennent la réflexion sont peu nombreux et répétitifs. La biographie de Michel Foucault fournit déjà à elle seule une batterie de prises de position que les chroniqueurs réutilisent sans modération tout au long de l'ouvrage. Sans que cela puisse remettre en cause la valeur intrinsèque de ces exemples (le GIP, l'affaire Croissant, les écrits de prisonniers, les ouvrages de Foucault, etc.), il faut bien avouer que l'ennui guette parfois le lecteur. Ennui qui touche à l'embarras à mesure que la répétition des mêmes exemples soutient de plus en plus difficilement l'éloge de l'événement entendu comme surgissement de nouveauté dans le réel. A répéter, même sous des points de vue décalés, le schéma critique de la généalogie et de la spécificité, on finit par essouffler les concepts

12. Les échos sont nombreux dans l'ouvrage. Ainsi des chapitres «Editer» et «Ecritures », ou encore «Usages et soulèvements », sans compter la récurrence des thèmes et des formulations rhétoriques, qui s'expliquent aussi sans doute par les provenances diverses des textes. 
et risquer le registre hagiographique. N'y a-t-il pas ici comme un écueil de l'incarnation ? Puisque le discours critique doit se fondre dans les objets qu'il prend (voilà le rôle de la reconnaissance de la spécificité), et non subsumer des faits sous des catégories plus générales (voilà ce qui prend les noms d'universaux), il peut finir par tourner sur lui-même comme une spirale sans fin, et ériger cette attitude en nouveau système d'interprétation tout aussi universalisant que les schémas qu'il dénonce. C'est bien là que se noue en dernière instance le paradoxe du discours du maître.

Du côté de Paul Veyne, l'hagiographie est évitée d'emblée par la forme même de l'ouvrage : centré à ce point sur le personnage que la ligne de force qui traverse le texte tend à s'en défaire. On joue la carte d'une écriture provocante et amusée à laquelle les divers ouvrages de l'historien de l'antiquité gréco-romaine nous ont habitués. Foucault est ici un ami, et la personne doit éclairer la pensée. C'est pourquoi il faudrait une étrange perversité pour que le texte se fasse épopée sainte (ce qu'Artières et Potte-Bonneville n'évitent pas toujours) : le sujet en est trop proche pour cela. Diverses remarques discrètement (ou parfois plus massivement) distillées touchant à la vie privée de l'homme Foucault cherchent à dessiner les contours du style Foucault. Une amicale tendresse enveloppe les pages d'une atmosphère intimiste, et il s'en faudrait de peu que le lecteur ne se sente transporté dans le monde de Diogène Laërce, dont les Vies et sentences des philosophes illustres résonnent du même entrain, celui de la joie de la vie philosophique. Que l'on ne se trompe pourtant pas: Veyne ne quitte jamais son cheval de bataille, et la douceur n'empêche pas la vigueur. A travers onze chapitres, l'auteur se donne pour objectif de dissiper tous les malentendus auxquels l'œuvre de Foucault a donné lieu, tant parmi les héritiers que les contempteurs. C'est que "Foucault n'a jamais exposé de pied en cap sa doctrine, il a laissé à ses commentateurs cette tâche redoutable 13 ».Veyne se charge donc de restaurer la pensée de son ami, de la même manière, selon lui, que Foucault aimait à restaurer tous les systèmes de pensée du passé. Il n'est pas ici directement question de reprendre un héritage ou de se déclarer «passeur » d'une œuvre d'avenir. L'enjeu est une certaine précision scientifique jamais trop appuyée, mais abondamment illustrée, tant par des citations que des exemples, sans compter les formules savoureuses. Il s'agit avant tout d'éclairer ce que l'auteur nomme le scepticisme ou encore «perspectivisme» de Foucault. Comme le titre l'indique, c'est en fait une vie pensante qui se déroule sous nos yeux, une vie pensante dont Veyne se fait le connivent doxographe.

Les cinq premiers chapitres développent les bases de la méthode critique foucaldienne. Recherche de "l'ultime différence individuelle ", critique des universaux, scepticisme méthodologique et vérité du scepticisme, ce chiasme axiomatique selon lequel la seule vérité stable s'énonce ainsi : toute vérité est 
devenue, et n'est qu'un discours de vérité. Ce que Paul Veyne explique plaisamment : en critiquant toute la «boutique des vérités " (c'est-à-dire les suites de vérités de l'histoire humaine), Foucault ne ruine pas la vérité, mais en assure une seule, la vérité critique, au prix de la ruine de toutes les autres, celles qui prétendent à la légitimité. "Constater que les éléments d'un bilan, considérés un par un, sont ruineux, comme le fait Foucault, ne ruine pas ce sombre bilan lui-même; bien au contraire, cela le confirme, le bilan et la boutique étant deux choses différentes, et ce bilan étant ruineux, à n'en pas douter ${ }^{14}$. » $\mathrm{Ou}$ encore, "un bilan ruineux ne s'emporte pas lui-même, le doute ne s'emporte pas lui-même 15 ». Manière théorique de répondre au paradoxe du maittre, mais pas encore pratique : en asseyant intellectuellement le scepticisme de Foucault, Veyne ne prend plus en compte le problème proprement corporel, pratique, indécollable de soi, du discours; problème dont Artières et Potte-Bonneville rendent très bien compte, tant et si bien qu'ils s'en trouvent empêtrés, pourrait-on dire, c'est-à-dire contraints à la répétition. Le sixième chapitre continue le développement spéculatif de ce thème sous l'égide de l'essence de la vérité heideggérienne critiquée par Foucault. Si pour Heidegger la non-vérité est une errance, c'est que toutes nos vérités sont des vérités de surface et que l'essence de la vérité nous est occultée. Par conséquent, toutes nos vérités ne sont que des discours superficiels ${ }^{16}$. Dès lors, il s'agit de questionner «l'essence de la vérité » elle-même. A l'inverse, pour Foucault, le bilan tragique doit laisser place à un travail critique positif, et non à une méditation sur l'essence de la vérité et le destin historial du Dasein. C’est pourquoi Veyne montre par la suite que le bilan sceptique ruineux de Foucault est précisément sa gloire. Veyne voit en Foucault et sa démarche le mouvement d'entière acceptation du vide de nos certitudes qui conduit, par un travail sur soi, à un amour renouvelé de ce que recèle le réel, à une passion de la positivité, qui ne se sépare pas de la lutte face à l'intolérable du moment présent. Voilà le " perspectivisme ». Ainsi Veyne signale-t-il : "Il n'y a pas de problèmes qui traversent les siècles, l'éternel retour est aussi un éternel départ (il aimait ces mots de René Char) ${ }^{17}$ ». C'est ce que, de leur côté, Artières et Potte-Bonneville thématisent avec précision comme la pensée engagée spécifique de Foucault, dont la particularité est de refuser la généralisation pour toujours préférer la chronique du temps présent. Deux thèmes différents, le scepticisme et l'engagement, qui se répondent avec bonheur d'un livre à l'autre.

Le livre commémoratif et combatif de Veyne transporte le lecteur dans l'intérieur même de la personne de Foucault. Parfois si près qu'il en devient difficile

14. Ibid., p. 71.

15 . Ibid., p. 112.

16. Heidegger M., Vom Wesen der Wabrheit, Francfort, Ed. Klostermann, 1954, p. 22 : « Ce que l'on est habitué à reconnaître, couramment aussi bien qu'en philosophie, comme erreur, la non-justesse (Unrichtigkeit) du jugement et la fausseté des connaissances, n'est que la sagesse superficielle de l'errance/erreur (Irrtum) ». Notre traduction.

17 . Veyne P., op. cit., p. 158. 
de faire la part des choses entre le vécu personnel de l'auteur avec son ami et les thèses développées. Ne dit-on pas que la biographie du philosophe est ce qu'il faudrait occulter pour mieux comprendre ? Paul Veyne montre justement que la biographie et la vie de Michel Foucault se mêlent profondément à sa pensée, comme ses textes sont un écho de ses expériences vitales profondes aussi bien que communes ${ }^{18}$. Combien la pensée de Michel Foucault est une pensée du présent qui se laisse convoquer par ce qu'il y a à faire, avant tout choix effectué a priori. L'universel doit se noyer dans l'histoire : les décisions ne se prennent pas sous les rassurantes auspices de vérités jugées éternelles. C’est sans doute aussi ce qu'Artières et Potte-Bonneville ont voulu montrer à travers leurs textes passionnés, commentant avec une grande implication le thème de la vie philosophique de Foucault, au sens pleinement classique : vivre la pensée. C'est aussi pourquoi aborder Michel Foucault conduit toujours à une remise en cause de son geste de lecteur ou de locuteur, puisque son ethos de magister est telle qu'elle a le pouvoir d'envoyer loin d'elle son élève. On peut y voir une ligne de crête, qui fera toujours du sceptique un être à la fois inimitable et dont les gestes appellent pourtant une authentique imitation, pour peu que l'on attache quelque prix à son enseignement. Sur cette délicate ligne de crête, Veyne a choisi l'amitié, Artières et Potte-Bonneville la transmission. Au lecteur d'en faire bon usage.

18. Les exemples abondent dans lesquels les anecdotes communes sont prises comme un éclairage pertinent d'un texte, d'une thèse ou d'une attitude théorique. Et cela motive assurément la lecture. 